\title{
Ferrofluid Hydrodynamics: waves, Jets and Free Drops
}

\author{
H. E. Potts and D. A. Diver \\ Dept. of Physics and Astronomy, Kelvin Building, \\ University of Glasgow, Glasgow G12 8QQ, Scotland, UK \\ email:hugh@astro.gla.ac.uk
}

Received on 17 January, 2001

\begin{abstract}
A novel approach to hydrodynamical studies uses the bulk force properties of magnetic liquids to probe the dynamics of (i) freely suspended drops; and (ii) unstable surface waves and jets. The fluid dynamics are imaged by a fast CCD camera, allowing thorough analysis of these timedependent phenomena. Ferrofluid drops are freely suspended in air by using magnetic fields to create an attractive force opposing gravity. The suspended drop then undergoes forced oscillations by perturbing the supporting magnetic field, and exhibits high order nonlinear modes of oscillation which can be driven until the drop bifurcates. Fluid surface waves and jets are investigated in cylindrical geometry. Nonlinear waves are magnetically driven, resulting in dramatic jets when the critical amplitude is exceeded. Such jets are observed to have a maximum acceleration exceeding $70 g$.
\end{abstract}

\section{Introduction}

A ferrofluid (FF) is a stable, colloidal suspension of sub-micron sized single domain magnetic particles in a liquid carrier, usually a light hydrocarbon solvent, an ester or simply water [1]. A ferrofluid in a magnetic field, experiences a force per unit volume given by:

$$
\mathbf{f}=\mu_{0} M \nabla H_{0}
$$

where $M$ is the magnetisation of the fluid, and $H_{0}$ is the applied magnetic field. The above equation assumes that the applied field is varying much more slowly than the magnetisation relaxation time for the ferrofluid $\left(\sim 10^{-7} \mathrm{~s}\right)$. In this case $\mathbf{M}$ is parallel to $\mathbf{H}_{0}$, and the ferrofluid behaves like a paramagnet, closely following the Langevin law. This forces the FF to move to the region of strongest magnetic field, offering a straightforward means of manipulating the fluid.

The aim of this paper is to demonstrate how ferrofluids can be used as a medium in which to study purely hydrodynamic systems. The volume force described above is used as a non mechanical mechanism to manipulate the fluid, allowing systems to be studied which would not be possible by conventional means.

In Section II we study of the dynamic behaviour of freely suspended ferrofluid drops, and Section III examines non-linear surface waves and jets excited by magnetic forces.

\section{Suspension of fluid drops}

There is a long history to the problem of the dynamics of the freely suspended drop, covering both experiment and theory: [2]-[8]. In this article, novel experiments large (5-8 $\mathrm{mm}$ ) drops of magnetic liquid are suspended by an actively controlled magnetic field gradient, which supports the drop against gravity. By applying a small time-dependent purturbation to the supporting magnetic field, the drop can be forced to oscillate. The equilibrium shape of a free, magnetised drop is approximately ellipsoidal [6], due the balance between the magnetic and surface tension energies (see [9] for further analysis).

\section{II.1 The free suspension}

In order to support a droplet of ferrofluid against gravity, the following condition must be satisfied:

$$
\rho \mathbf{g}=\mu_{0} M \nabla H_{0}
$$

where $\rho$ is the mass density of the ferrofluid.

The practical implementation of (2) requires a coil arrangement designed to confine the drop in the horizontal plane, with vertical stability achieved via an active feedback system. In Fig.1 a cross section of the coil shape used with contours of field strength is shown. Information on the drop position was obtained from a linear array of 16 photodiodes with a spacing of $1 \mathrm{~mm}$. These measured the shadow cast by the suspended drop from a diffuse infra-red linear light source. The output 


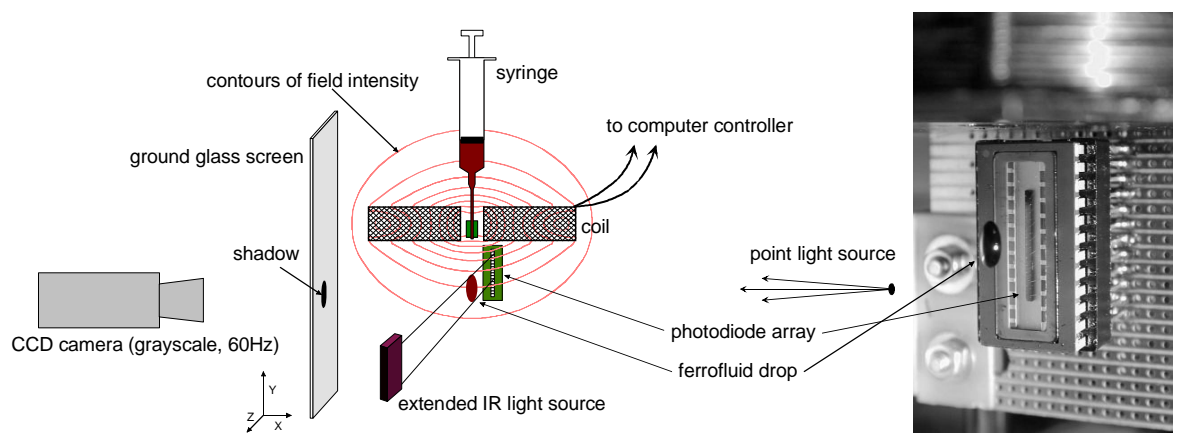

Figure 1. Drop support coil with magnetic field shape and imaging arrangement.

from these was passed to a control computer that could calculate the drop position and vertical axis length with a resolution of about $0.1 \mathrm{~mm}$. To stabilise the drop in the vertical direction the drop position information was fed into a software implemented proportionalintegral-derivative (PID) controller which set the current through the coil of the levitation magnet. Typical field strengths were around $0.02 \mathrm{~T}$. A drop of FF was launched from a syringe into the apparatus, where the PID controller detected it, and adjusted the field to catch and hold it. The volume of the drop dispensed was controlled by the diameter of the needle. A photograph of a stably suspended drop is shown in Fig. 1; the equilibrium shape is clearly elliptical in cross-section. Images of the drops were obtained using a distant pinhole light source to cast a shadow of the drop onto a ground glass screen. This was then recorded using a fast CCD camera interfaced to the controller computer

\section{II.2 Forced oscillations}

The effect of a sinusoidal disturbance of the current in the stabilising coil is to add a ripple to the potential well in which the drop is sitting, causing the drop to oscillate. The predominant effect is the alteration in the ambient magnetic field, which in turn changes the drop shape via changes in the magnetic tension at the surface of the drop. For sufficiently high driving frequencies ( $>10 \mathrm{~Hz}$ for average sized drops) the position of the centre of mass of the drop was essentially unchanged. To investigate the form of high amplitude oscillations the drop was driven at resonance, i.e. the frequency at which the drop response was maximal for a given amplitude input.

A wide range of oscillation amplitudes were possible, from very small amplitudes for which the drop response was essentially linear, to large perturbations in which nonlinear modes were evident. The left hand image in Fig. 2 shows camera frames of a small amplitude case in which the equilibrium ellipticity was 0.80 , and the drop volume was $16 \mathrm{~mm}^{3}$. The drop oscillates happily in a prolate spheroidal mode at a frequency of $26 \mathrm{~Hz}$. Note that as the camera can only capture frames at $60 \mathrm{~Hz}$ the frames presented in Fig. 2 are de-aliased data from a much longer continuous time series.

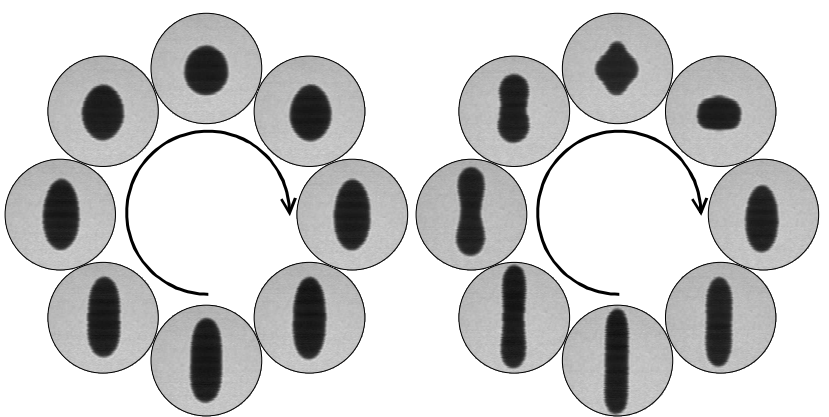

Figure 2. Oscillation of a drop shown as de-aliased data over a cycle. The left hand images show the drop driven at low amplitude at resonance at $26 \mathrm{~Hz}$. The right hand images show the same drop driven at high amplitude. The resonant frequency has now dropped to $23 \mathrm{~Hz}$. The ferrofluid was Ferrofluidics [10] EMG909 and the drop volume was $16.0 \mathrm{~mm}^{3}$.

The same drop was then forced at a larger driving amplitude, causing significant nonlinearity in the response. The camera images shown in in the right hand cycle (de-aliased as before) reveal a greater elongation of the drop, with the evolution of the profile showing clear nonlinearity and the onset of higher-order modes. Note that as the amplitude of the driving perturbation was increased, the resonant frequency of the drop was observed to reduce, to $23 \mathrm{~Hz}$ in this case.

If the drop is forced beyond a critical amplitude it eventually bifurcates, as shown in the real-time frame sequence in Fig. 3. Note that as this is not a periodic event, de-aliasing is not possible. The frames show spectacular higher-order modes in the evolution towards splitting. Frames 3,6 and 9 show similar modes to those seen in Fig.2, albeit at significantly higher amplitude. Note that frame 6 almost certainly has concave top and bottom surfaces hidden from view since these frames are only projections and not cross-sections. Frame 12 shows the formation of a liquid bridge as the drop begins to split, but ultimately reforms. In frame 


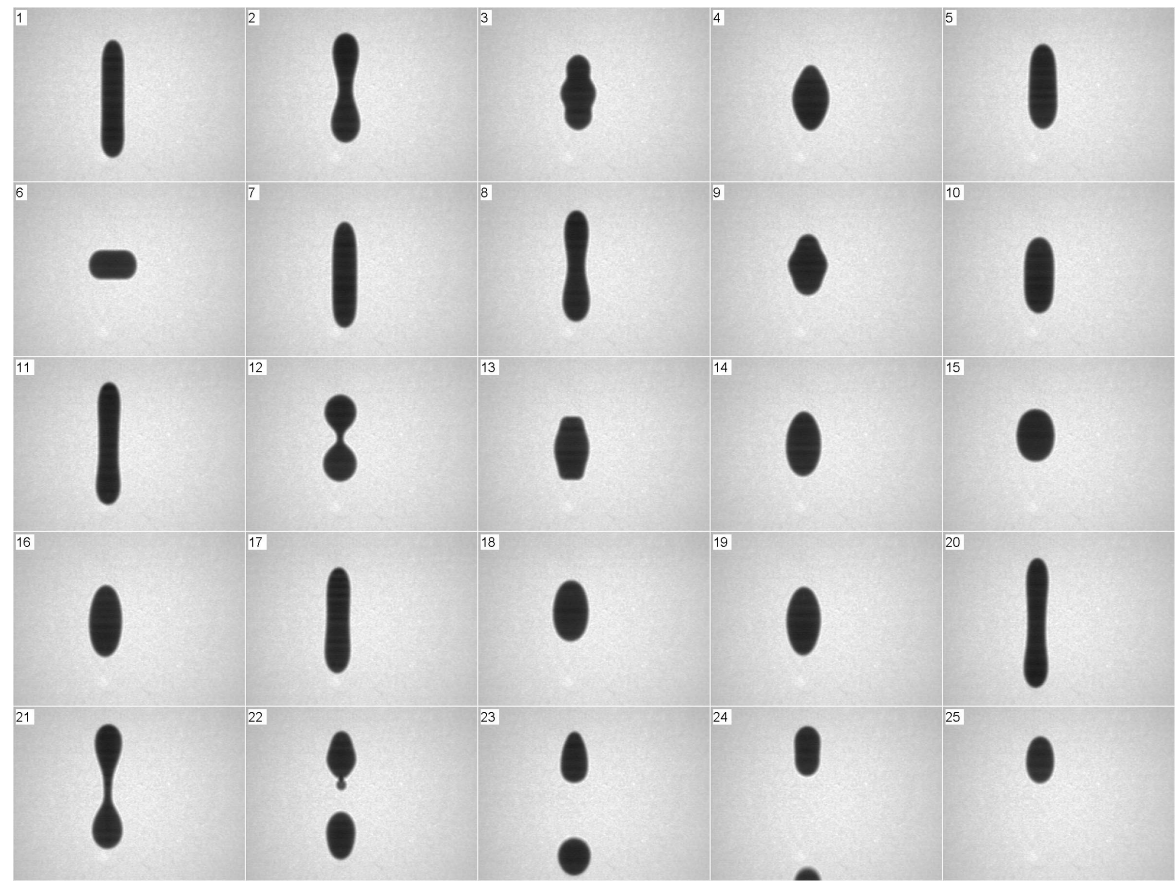

Figure 3. Consecutive frames at $(16 \mathrm{~ms})$ intervals showing a ferrofluid drop driven to bifurcation.

\section{II.3 White noise stimulation}

In order to explore its full frequency response, a suspended drop was stimulated by white noise at a $1 \mathrm{kHz}$ sample rate and several amplitudes via the control coil. Even at high driving amplitudes, the energy content in a single frequency is small, and the drop dynamics remained close to linear. The Fourier transform of the resulting response is given in Fig.4, in which the fundamental oscillation frequency at around $28 \mathrm{~Hz}$ is the most prominent, and with at least one higher order mode visible in the region of $88 \mathrm{~Hz}$. There is also a feature visible at $56 \mathrm{~Hz}$, which is not a true fundamental mode; rather it is a harmonic of the non-sinusoidal fundamental. Note also the slight drift downwards in frequency of the fundamental mode at higher driving amplitudes.

\section{Surface wave studies}

The concept of a maximum amplitude standing wave has long been appreciated experimentally, and theoretical analysis of the shape of the one-dimensional maximum wave, [12], [13] agrees with the experiment in that they each recover a maximum angle at the crest of a standing wave to be $90^{\circ}$, with the theoretical assumption that the maximum vertical acceleration associated with this critical wave is equal to $-g$, the acceleration due to gravity.
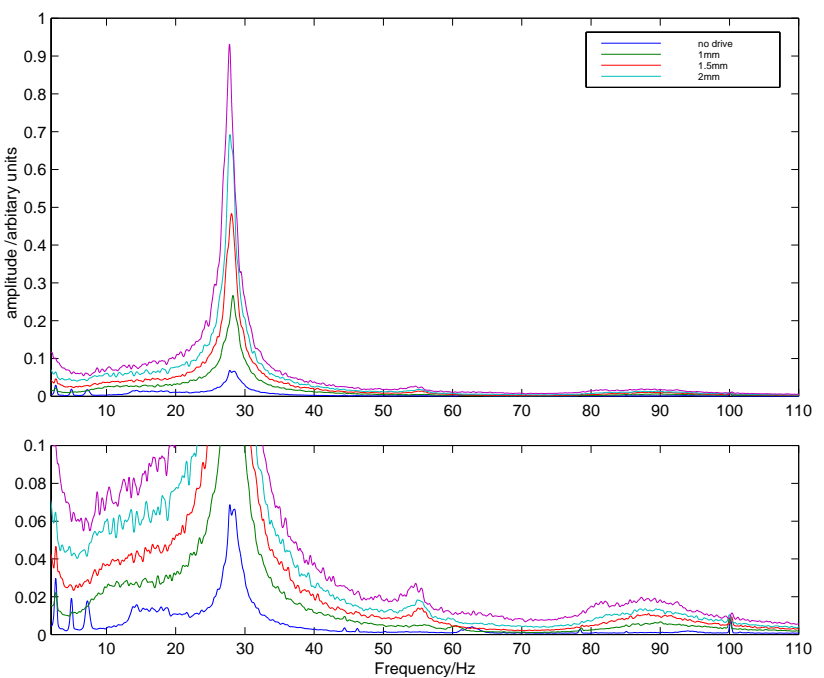

Figure 4. Frequency response of a ferrofluid drop of volume $17 \mathrm{~mm}^{3}$ and rest ellipticity 0.81 , subjected to white noise driving signal at different amplitudes. The lower plot shows more detail around the resonances. The response for no driving signal is due to noise in the PID controller system.

Exceeding the maximum steepness of a standing wave eventually results in fluid jetting or splashing. A comprehensive review of the topic is given by Peregrine [14], where mathematical models are developed. 
Numerical simulations of splashing [15] agree closely, and very recent experimental studies, [16] offer further insight into the rôle of singularities and bubbles in the evolution of jets from over-forced standing surface waves.

\section{III.1 The Experimental Setup}

The fluid is driven by a coil wrapped around a cylindrical vessel of internal diameter $86 \mathrm{~mm}$, as shown in Fig. 5 .
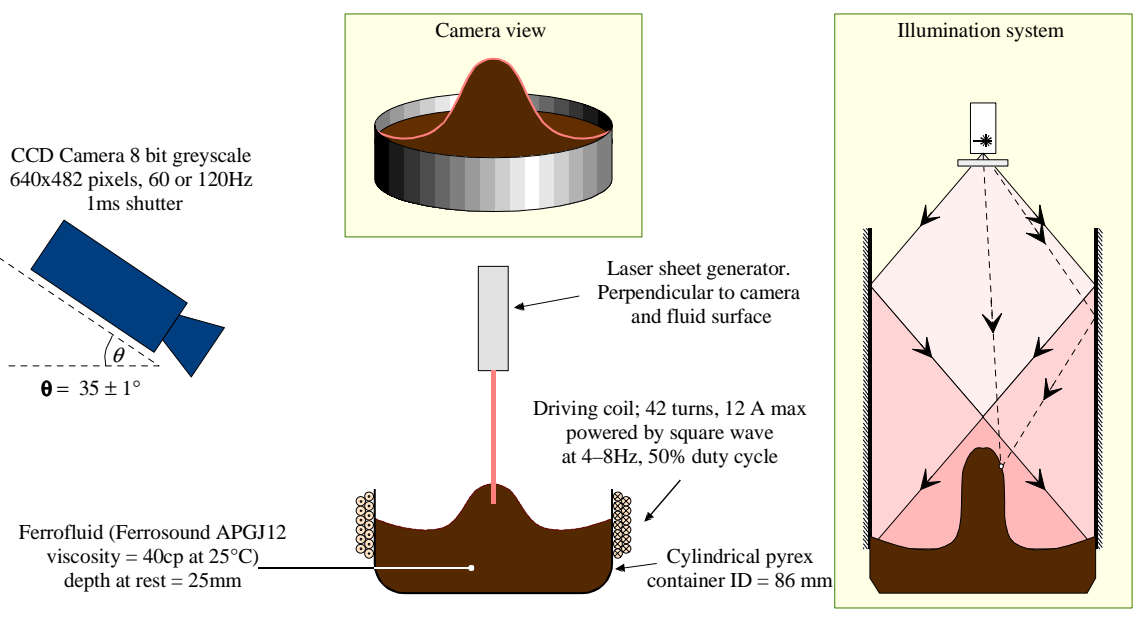

Figure 5. Experimental setup.

The magnetic field produced by the coil has a gradient at the vessel edge, which pulls the FF outwards and upwards, as described by (1). For all experiments, the FF used was Ferrofluidics APGJ12, and the temperature of the fluid was maintained at $32 \pm 2^{0} \mathrm{C}$ in order to keep the viscosity constant. The fluid was then excited over a range of frequencies with a $50 \%$ duty cycle square wave. As losses from the system were small, the required driving force was also small, and had only to be sufficient to compensate for viscous losses. Hence the square wave driver applied to the resonant system did not excite significant high harmonics.

Imaging the fluid surface presented considerable technical problems, given that FF is black and very opaque, somewhat like used engine oil. The diffuse reflections are therefore very weak, and the specular reflections relatively bright. To get a profile of the axially symmetric surface produced by the surface waves, the whole arrangement was lit with a vertical sheet of laser light, aligned perpendicular to the camera.

The fluid motion was captured using a fast progressive scan CCD camera, and the images were streamed into computer memory by a frame grabber. Frames could be gathered at a rate of $60 \mathrm{~Hz}$, at a resolution of $640 \times 482$ pixels with 256 levels of grey scale, or at twice the frame rate with half the vertical resolution. The camera was angled at $35^{0}$ to the horizontal to allow it to see into the bottom of the surface wave troughs. As a result, the images obtained were vertically compressed, and distorted by perspective. Imaging a square grid in the plane of the laser light allowed the precise nature and extent of this distortion to be measured, allowing the images to be corrected.

This experimental setup produced images consisting of a dim line from the diffuse surface reflections, and various bright specular reflections from spurious sources. The surface data was extracted automatically from the images using a computer algorithm that searched for the characteristic line profile, using information from previous frames to predict its likely position and profile. Examples of surface wave profiles recovered by this technique are shown in Fig. 6 .

\section{III.2 Results}

The experimental observations are presented here, together with analysis of the data, and some simple modelling. Two aspects of the driven fluid were explored: resonant standing waves, and wave breaking and jetting.

\section{III.2.1 Surface wave response as function of driv- ing frequency}

Here the FF was subjected to a driving field of low amplitude, for various different frequencies. The resonant response at $4.41 \mathrm{~Hz}$ is clearly seen in Fig.6, together with a further resonance near $6.2 \mathrm{~Hz}$. A further weak resonance around $(7.9 \pm 0.5) \mathrm{Hz}$ was also observed, and has been included for completeness. Note that at resonance, the waves are finite amplitude, and well below the threshold for jetting. Because of the camera 
angle in the imaging system as arranged for this particular experiment, the edges of the vessel at the wave height cannot be viewed, and so the profiles of the fluid in Fig. 6 do not show the full width of the disturbance; instead the axes have been extended to indicate the true width of the vessel. However, since the prime goal here was to show wave resonances as a function of driving frequency, this is not a significant drawback, particularly since the jetting experiments concentrate on the fundamental mode. Clearer data for this $4.4 \mathrm{~Hz}$ fundamental mode are shown in Fig.8, where the full width of the disturbance is presented.

A graph of the wave amplitude in response to the driving frequency is shown in Fig.7, demonstrating clearly the resonances at $4.4 \mathrm{~Hz}$ and $6.2 \mathrm{~Hz}$. Note that the resonances are relatively wide, and asymmetric, reflecting the fact that the fluid is viscous, allowing offresonance coupling, and the waves being excited at resonance are finite amplitude. Unfortunately, the resonance at $7.94 \mathrm{~Hz}$ has poor signal-to-noise ratio when displayed in this graph, but it is observed experimentally, and therefore worth quoting.
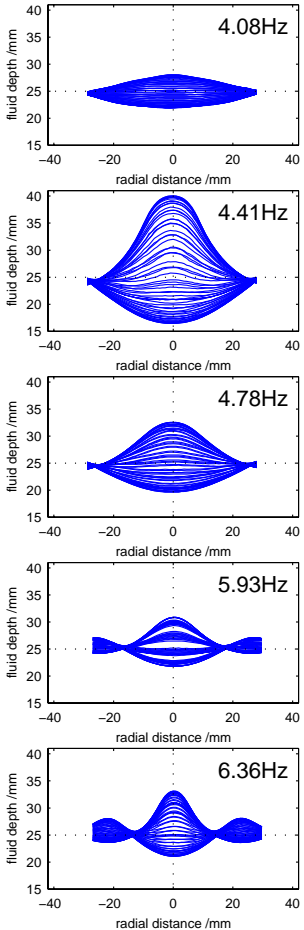
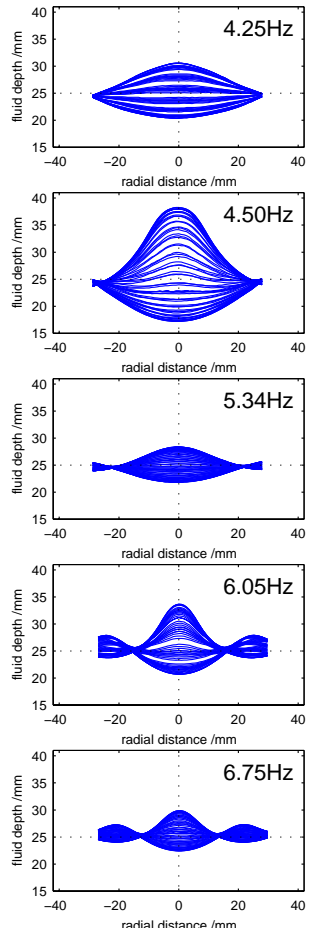
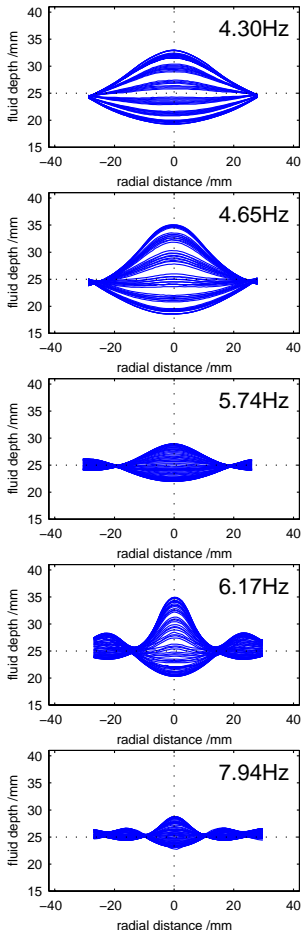

Figure 6. Experimentally recovered wave profiles for low amplitude oscillations at different frequencies. True vessel diameter is indicated by the extent of the horizontal axis.

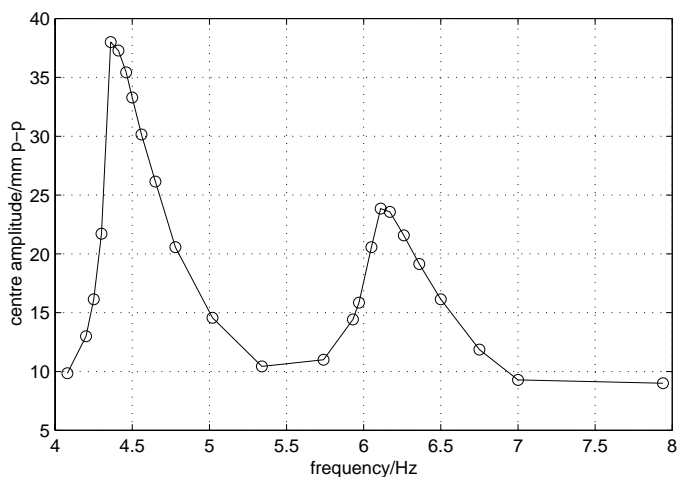

Figure 7. Plot of amplitude response as a function of frequency, showing the first 2 resonances. Note that a weak resonance was also observed at $7.9 \mathrm{~Hz}$.

The solution for the wave profile for cylindrically symmetric water waves [17] is $\sin (\omega t) J_{0}(k r)$, where $J_{0}$ is the Bessel function of order $0, r$ is the radial coordi- nate, $\omega$ is the wave frequency $k$ is the wavenumber and $\omega^{2}=g k$. Taking the small-amplitude wave frequency to be at the centre of each resonance in Fig. 7 yields a satisfactory fit to this linear theory.

III.2.2 Resonant surface wave response as function of driving amplitude

In this set of experiments, the amplitude of the driving field was varied at the frequency of the lowest resonant mode (at around $4.4 \mathrm{~Hz}$, allowing for the fact that the resonant frequency drops as the wave amplitude increases). Typical responses for low and high amplitude forcing are presented in Fig. 8. 


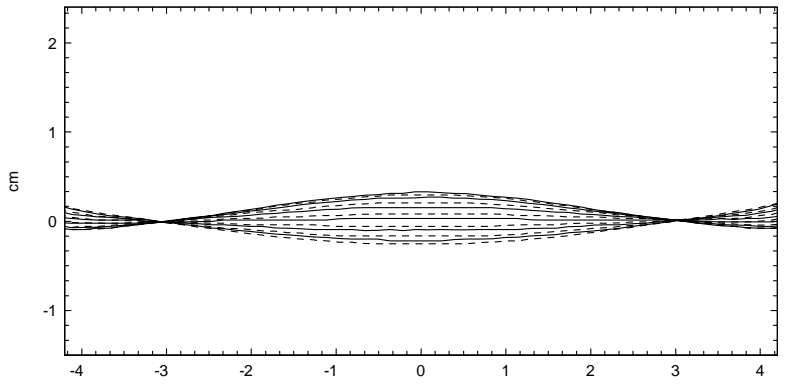

$\mathrm{cm}$

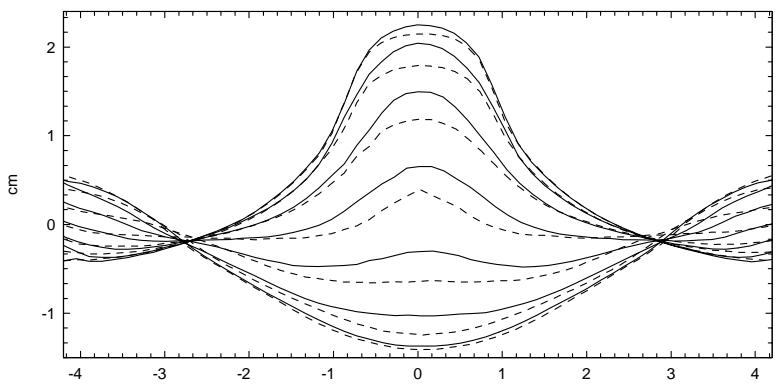

$\mathrm{cm}$

Figure 8. Experimentally recovered wave profiles for low (top) and high (bottom) amplitude oscillations for the lowest frequency mode (around $4.4 \mathrm{~Hz}$ ). Dotted lines indicate the profile when the peak is rising; solid lines when the peak is falling.

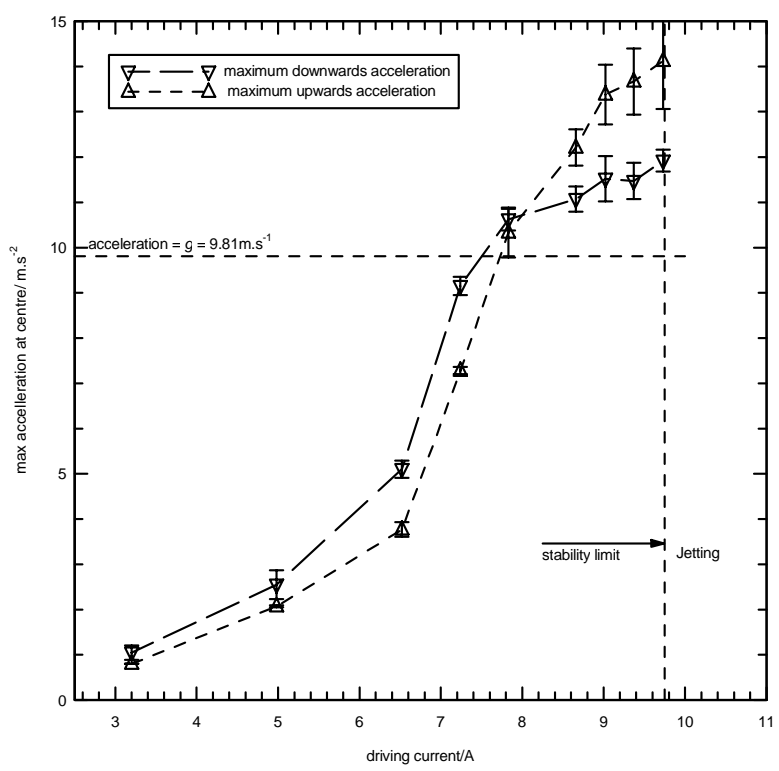

Figure 9. Acceleration at the crest as a function of driver amplitude.

In the theoretical descriptions [12], the maximum downward acceleration at the crest of the surface wave must not exceed that due to gravity. In Fig.9, the measured acceleration of the crest as a function of applied amplitude, driven resonantly, is plotted. Experiment shows that jetting begins if the driving current exceeds $I_{\max }=9.7$ A. For low amplitude waves, the downward acceleration of the crest is less than the upward, but for high amplitude waves this trend is reversed as the slope of the downward acceleration curve decreases markedly, and tends to a value of just under $12 \mathrm{~m} \mathrm{~s}^{-2}$ at threshold. Clearly, this maximum downward acceleration exceeds the theoretical maximum by $20 \%$, and so there must be another downward force in addition to gravity. This extra force is provided by the surface tension, based on applying the Young-Laplace equation to the measured radius of curvature of the wave crest (see [18] for details).

\section{III.2.3 Surface Jets}

Choosing the lowest harmonic at around $4.4 \mathrm{~Hz}$, the amplitude of the standing wave was increased to beyond the maximum amplitude, so that the current in the coil exceeded $I_{\text {max }}$. The fluid response is shown in Fig.10 as a subset of 15 frames, selected from a complete sequence covering a $633 \mathrm{~ms}$ period. The early frames show the extent of the nonlinearity in the fluid motion, with the profile flattening very clear at $233 \mathrm{~ms}$. At $417 \mathrm{~ms}$ an extraordinarily fine jet of $0.2 \mathrm{~mm}$ diameter is seen to erupt from an otherwise relatively flat surface, and is a precursor to a much more substantial jet evident some $50 \mathrm{~ms}$ later. Taking the surface speed to be approximately zero at the centre at $400 \mathrm{~ms}$, the precursor jet has travelled a vertical distance of approximately $105 \mathrm{~mm}$ in the $17 \mathrm{~ms}$ prior to the next frame, which equates to an average acceleration of $726 \mathrm{~m} \mathrm{~s}^{-2}$, with a corresponding maximum speed of $12.3 \mathrm{~m} \mathrm{~s}^{-1}$. In common with Zeff et al [16], we take the initiation of the jet to be a singular event, and neglect gravity.

Jetting occurs because the surface tension is broken by the appearance of a sharp feature, associated with the flattening of the surface wave profile in the cycle immediately before the onset of instability.

Fig. 11 shows this effect very clearly: not only has the wave top flattened, but a dimple has appeared in the centre. When the main oscillation moves into its downward stroke, the edges of the dimple meet in a sharp feature that destroys the surface tension, leading to the eruption of a jet from this singular event. 


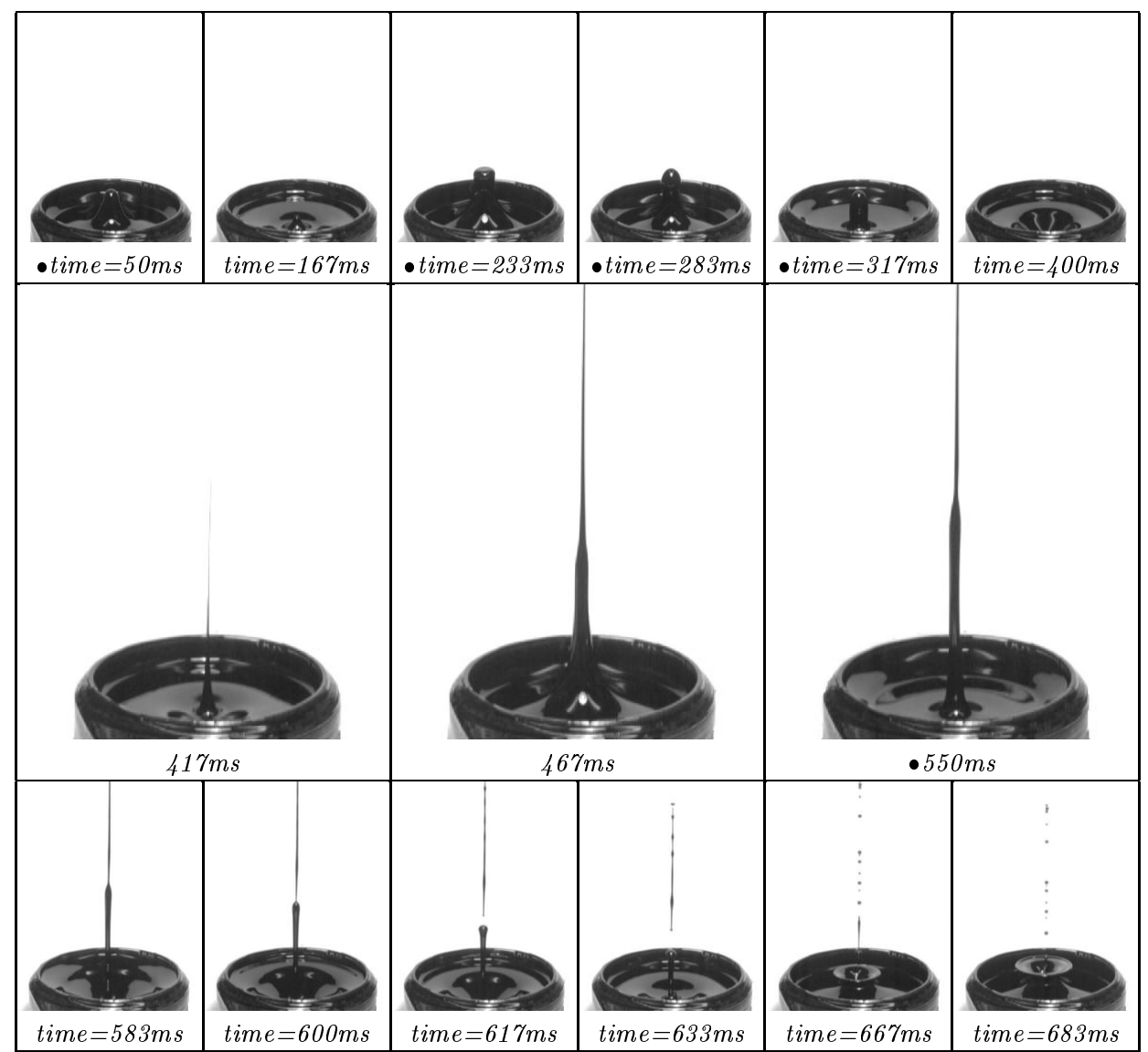

Figure 10. Sequence of frames showing a rapidly moving jet evolving from an overforced standing wave; • denotes non-zero coil current.

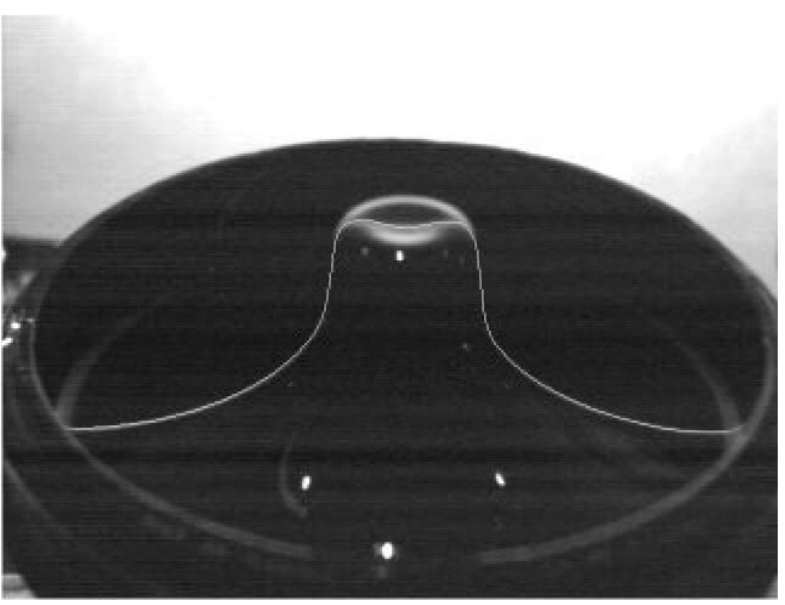

Figure. 11 Evolution of a sharp feature on an unsteady overdriven oscillation. The laser line has been enhanced for clarity.

\section{Conclusions}

We have demonstrated the efficacy of magnetic liquids as tools in the investigation of non-linear hydrodynamical phenomena.

In this paper we have shown that it is possible to support large drops of ferrofluid in a magnetic field us- ing an active control system. These suspended drops can then be manipulated by applying ]arbitrary timevarying signals to the supporting potential, allowing drops to be studied as if in microgravity. The equilibrium shape as a function of applied magnetic field was verified for suspended drops. For small amplitude oscillations, ellipsoidal (or more correctly, prolate spheroidal) modes are observed. Quantitative measurements of the mode frequencies were undertaken using broad-band white noise stimulation.

This paper also shows how magnetic liquids can be used to study surface waves for even small volumes of a viscous liquid (in this case, $145 \mathrm{cc}$ ). The wave evolution as a function of forcing amplitude was investigated, resulting in the creation of rapidly accelerating jets which were imaged by a fast, high resolution CCD camera. This combination of magnetic forcing and fast image acquisition has allowed the surface instability and consequent jet evolution to be studied in great detail. 


\section{Acknowledgements}

The authors are grateful for research funding from the UK EPSRC, under grant number GR/L90699. Thanks are also due to Richard Barrett, Sinclair Bremner, Paul Millar and Neal Wade for stimulating discussions.

\section{References}

[1] Rosensweig, R.E.: 1985,Ferrohydrodynamics, Cambridge University Press, Cambridge, UK.

[2] Azuma, H., Yoshihara, S., J. Fluid Mech. 393, 309 (1999).

[3] Lee, C.P., Anilkumar, A.V., Hmelo, A.B., Wang, T.G., J. Fluid Mech. 354, 43 (1998).

[4] Lebedev, A.V, Morozov, K.I., JETP Lett. 65, 161 (1997).

[5] Bacri, J.-C., Cebers, A., Perzynski, R., Phys. Rev. Lett. 72, 2705 (1994)

[6] Arkhipenko, V.I., Barkov, Yu.D., Bashtovoi, V.G.: Magnitnaya Gidrodinamika 3, 131 (1978).

[7] Séro-Guillaume, O.E., Zouaoui, D., Bernardin, D., Brancher, J.P., J. Fluid Mechs. 241, 215 (1992).
[8] Suryanarayana, P.V.R. and Bayazitoglu, Y., Phys. Fluids A, 3, 967 (1991).

[9] Potts, H.E., Barrett, R.K., Diver, D.A.: "Dynamics of freely-suspended drops", Journal of Physics D: Applied Physics, 34 2529, (2001).

[10] Ferrofluidics Ltd, Talisman Business Centre, Bicester, Oxon. OX26 6HR, UK.

[11] Taylor, G.I., Proc. Roy. Soc. Lond. A218, 44 (1953).

[12] Penney, W.G., \& Price, A.T., Phil. Trans. Roy. Soc. Lond. A244, 254 (1952).

[13] Longuet-Higgins, M.S., Proc. Roy. Soc. Lond. A331, 445 (1973),

[14] Peregrine, D.H., J. Fluid Mech. 106, 59 (1981).

[15] Anderson, A., Diver D.A., Peregrine, D.H. 1990, Proc 5th International Workshop on Water Waves and Floating Bodies, Manchester.

[16] Zeff, B.W., Kleber, B., Fineberg, J., Lathrop D.P., Nature, 403, 401 (2000).

[17] Stoker, J.J.: 1957, Water Waves, Interscience Publishers, New York, USA.

[18] Potts, H.E., Diver, D.A.: "Large amplitude ferrofluid surface waves and jets" New Journal of Phys. 3: 7.17.14, 2001, www.njp.prg. 